\title{
Electrical Conductivity Studies Of Pani-Flyash Blends
}

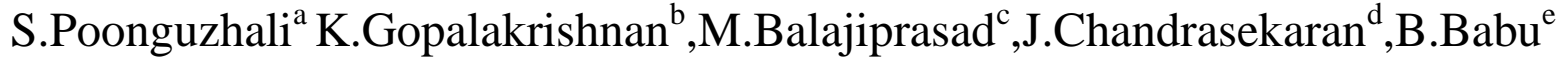 \\ ${ }^{a}$ Asso.Prof,Dept.of Electronics,S.N.R.SonsCollege,Coimbatore,India \\ ${ }^{b}$ Prof \& Deputy Controller, Bharath University, Chennai,India \\ ${ }^{c}$ Asso.Prof,Dept.of Electronics,,L.R.G.College,Tirupur,India. \\ ${ }^{d \gtrless e}$ Dept,of Physics,SRMV College,Coimbatore,India.
}

\begin{abstract}
The conducting polymers have conjugated double bonds in their backbone.There are many different types of conducting polymers. The most common conducting polymers are Polyacetylene (PA),Poly (phenylene), Polythiophene Polypyrrole,Polyaniline(PANI) etc., . The addition of FLYASH in PANI results in an enhancement of microwave absorption with an added advantage of industrial waste utilization. The PANI-FA compositeswere prepared with different concentration of flyash to aniline ratio (50:50,60:40,70:30,80:20).AC and DC conductivity studies were carried out for the blends using Four probe technique for various temperature.
\end{abstract}

Keywords: - PANI, FLYASH, Four probe technique

\section{INTRODUCTION}

Polymers in general are mainly used in electrical, optical and electronic devices as insulators because of their very high electrical resistivity. Conducting Polymers(CPs) have the electronic properties of semiconductors and mechanical parameters of polymers. Polyaniline (PANI) is one of the most promising conducting polymers because of its unique properties like ease of preparation in aqueous medium, good stability in air, simplicity in doping, improved electronic properties, excellent environmental stability, moderately high conductivity etc. Polyaniline (PANI) is one of the most common CPs, which can be synthesized through simple and relatively cheaper methods. Polyaniline is more noble than copper and slightly less noble than silver which is the basis for its broad use in printed circuit board manufacturing (as a final finish) and in corrosion protection. Polyaniline can be found in one of three idealized oxidation states.

- leucoemeraldine - white/clear \& colorless $\left(\mathrm{C}_{6} \mathrm{H}_{4} \mathrm{NH}\right)_{n}$

- emeraldine - green for the emeraldine salt, blue for the emeraldine base $\left(\left[\mathrm{C}_{6} \mathrm{H}_{4} \mathrm{NH}\right]_{2}\left[\mathrm{C}_{6} \mathrm{H}_{4} \mathrm{~N}\right]_{2}\right)_{\mathrm{n}}$

- $\quad$ (per)nigraniline - blue/violet $\left(\mathrm{C}_{6} \mathrm{H}_{4} \mathrm{~N}\right)_{\mathrm{n}}$.

On the other hand, Fly Ash (FA) which is the by-product of combustion of coal, are generally treated as waste materials. There is great challenge to use the waste of thermal power stations in the form of fly ash as reinforcement for the conducting polymers to be good dielectric materials. Less than half of the ash is used as a raw material for concrete manufacturing and construction. The remaining ash is directly dumped to land fill or simply piled up. To save our environment, new ways to utilizing the FA need to be explored. This paper discussed about the preparation of PANI-FA blends and the properties of ac and dc conductivity. The blending of FA in PANI introduces uniform porosity which will provide a great advantage in the field ofelectro magnetic shielding, gas sensor and bio sensor.

\section{EXPERIMENTS}

The Polyaniline and fly ash cannot be compress as a pellet without using any excipients. So wet granulation method was used to compress them as pellets. In wet granulation method a binding agent is required to mix polyaniline and fly ash. Poly Vinyl Pyrolidone (PVP) is the best choice of binder for mixing Polyaniline and fly ash for preparing pellets. The compression is performed using "Rimex minipress compression tablet punching machine" using $4 \mathrm{~mm}$ and $10 \mathrm{~mm}$ diameter punch (thickness found by micrometer). Required quantity of Polyaniline and fly ash are weighed using single pan balance - "Dhoona 200 D". Then required amount of PVP is added and mixed with one or two drops of water and is made as granules using sieve number 22. It is dried and compressed. At different ratios of polyaniline and fly ash, along with PVP, four different samples with various compositions were made as pellets and used for further investigations.
1.SAMPLE (A)
POLYANILINE(50\%)+FLYASH $(50 \%)$
2. SAMPLE (B)
POLYANILINE(60\%)+FLYASH $(40 \%)$
3. SAMPLE (C)
POLYANILINE(70\%)+FLYASH $(30 \%)$
4. SAMPLE (D)
POLYANILINE( $80 \%)+$ FLYASH $(20 \%)$ 
DC electrical conductivity studies of the four samples were performed on Keithley high resistance meter/electrometer $6517 \mathrm{~B}$. In this study, the sample was sandwiched between two copper electrodes and annealed in an oven. The temperature was controlled by a controller. The current was measured with respect to the applied voltage across the sample at different temperature and the sample which shows the good conductivity was noted down.

AC electrical conductivity - Dielectric studies of the samples were carried out using a HIOKI 3532-50 LCR HITESTER impedance meter. The studies were carried out at different frequency from $50 \mathrm{~Hz}$ to $5 \mathrm{MHz}$ at various temperatures. The change in conductivity for different samples and its dielectric properties were calculated using suitable formula.

\subsection{DC conductivity studies:}

\section{RESULT \& DISCUSION:}

The Dc electrical conductivity of the four samples was performed on Keithley high resistance meter/electrometer 6517B. In this study initially the current was measured with respect to the applied voltage across the sample at different temperatures. The sample was sandwiched between two copper electrode and annealed in an oven. Controller was used to control the temperature. The DC electrical conductivity has been calculated using the following formula:

$$
\Sigma=\left(\mathrm{I}^{*} \mathrm{~L}\right) /(\mathrm{V} * \mathrm{~A}) \text { where }
$$

I is the current. $\mathrm{V}$ is the voltage, $\mathrm{L}$ is the thickness of the sample and $\mathrm{A}$ is the cross sectional area of the sample.

Fig(1) (Voltage.vs. $\sigma$ DC) SAMPLE (A)

Fig(2) (Voltage.vs. $\sigma$ DC) SAMPLE (B)
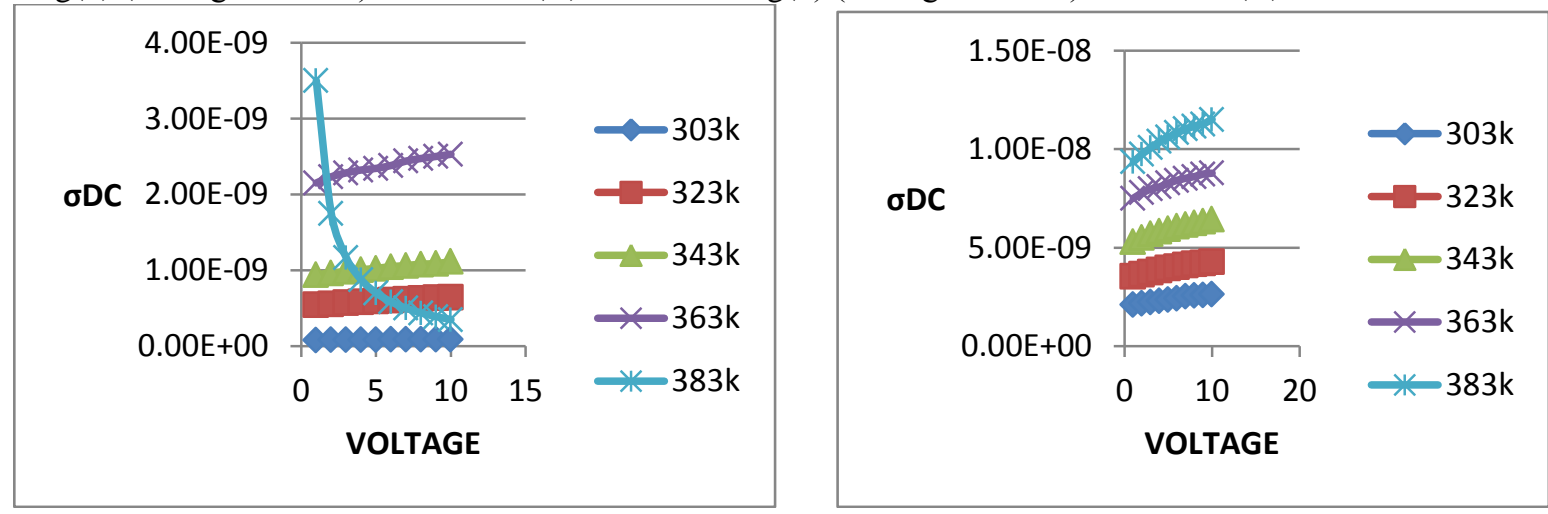

Fig(3) (Voltage.vs. $\sigma$ DC) SAMPLE (C)

Fig(4) (Voltage.vs. $\sigma$ DC) SAMPLE (D)
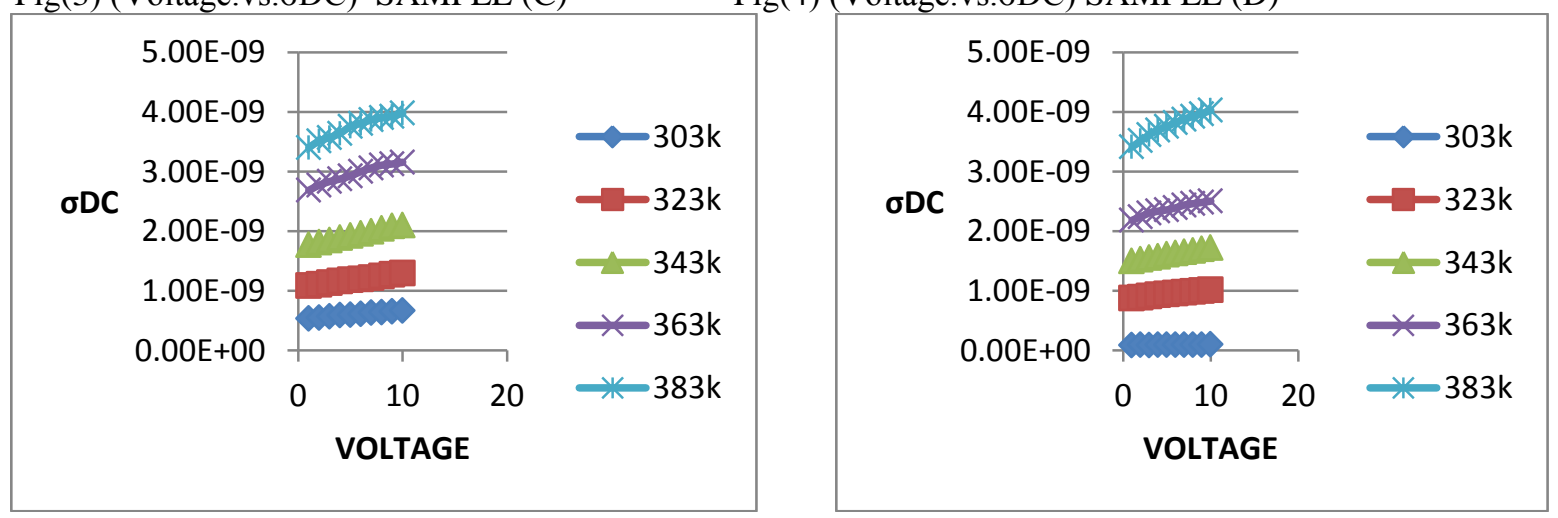

From the graph it shows that the DC conductivity increases with an increase in temperature. By comparing the graph, Fig (2) provides a linear increase in conductivity with the voltage. So the blend of PANI And Flyash in the ratio of 60:40 (sample B)percentage provides a good conducting property among all sample.

\subsection{AC Conductivity Studies:}

AC conductivity studies were carried out using HIOKI3532-50 LCR HITESTER impedance meter. The experiment was carried out at different frequencies from $50 \mathrm{~Hz}$ to $5 \mathrm{Mhz}$ at various temperature. The instrument provides the value of capacitance, impedance and dielectric loss for the four samples in the frequency ratio mentioned above. The dielectric constant was calculated using the formula, $\epsilon_{\mathrm{r}}=\mathrm{Cd} / \epsilon_{0} \mathrm{~A}$. Where 
$C$ is the capacitance and $d$ is the thickness, $A$ is the cross sectional area of the sample and $\epsilon_{0}$ is the free space permittivity of the sample. The AC conductivity was calculated using the formula, $\sigma_{\mathrm{ac}=} \mathrm{\epsilon}_{0} \mathrm{C}_{\mathrm{r}} \mathrm{D} \omega$.

Fig(5) (logf.Vs. $\left.\sigma_{\mathrm{ac}}\right)$ SAMPLE (A)

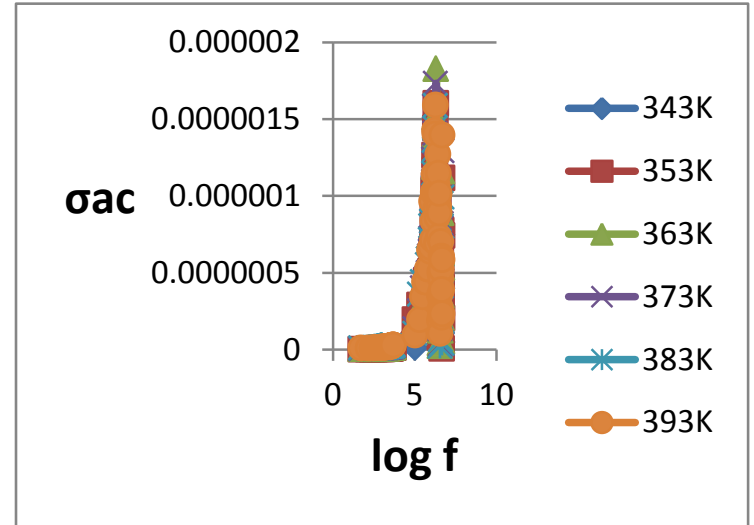

Fig(7) (logf.Vs. $\left.\sigma_{\mathrm{ac}}\right)$ SAMPLE (C)

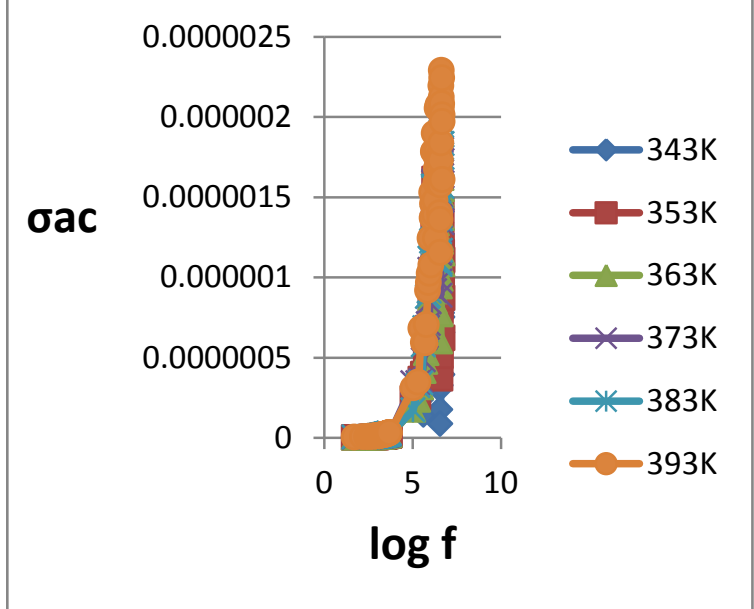

Fig(6) (logf.Vs. $\sigma_{\text {ac) }}$ SAMPLE (B)

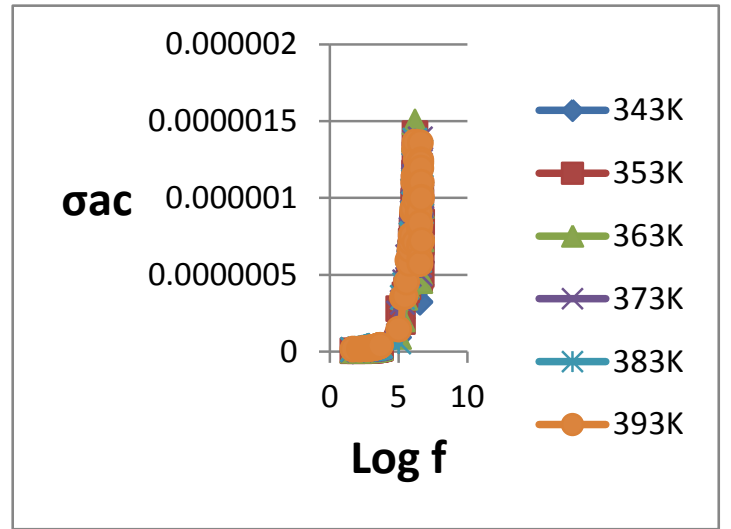

Fig(8) (logf.Vs. $\sigma_{\mathrm{ac}}$ SAMPLE (D)

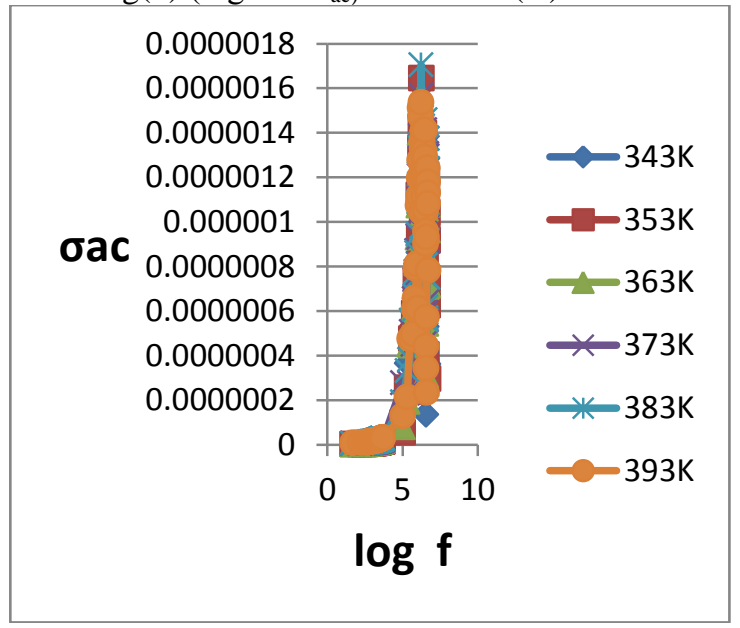

From the graph, sample(C) provides maximum conductivity and gives a linear increase of conductivity with an increase in temperature.

\section{CONCLUSION}

PANI and Fly ash blend was initially mixed with PVP to make as pellet for different samples, successfully. The DC and AC conductivity studies were carried out and the necessary graph was plotted. This study shows that the conductivity is lowered by the presence of FA. But the gradual increase of Fly ash does not provide a linear decrease of conductivity. From the graph sample (B) provides a good DC conducting property. By increasing the temperature, the increased $\mathrm{AC}$ conductivity provides a good response for sample $(\mathrm{C})$ among all. To conclude the electrical properties, FA can be used to control the conductivity of PANI to make it suitable for various applications.

\section{REFERENCES}

[1] S C RAGHAVENDRA, SYED KHASIM, M REVANASIDDAPPA,M V N AMBIKA PRASAD AND A B KULKARNI, Synthesis, characterization and low frequency a.c. conduction of polyaniline/fly ash composites, Bull. Mater. Sci., Vol. 26, No. 7, December 2003, pp. 733-739. ( Indian Academy of Sciences.

[2] Raju Khan, Puja Khare, Bimala Prasad Baruah, Ajit Kumar Hazarika, Nibaran Chandra DeySpectroscopic, Kinetic Studies of Polyaniline-Flyash Composite, Advances in Chemical Engineering and Science, 2011, 1, 37-44

[3] Avanish Pratap Singh,1,2 Anoop Kumar S.,1 Amita Chandra,2 and S. K.Dhawan1, Conduction mechanism in Polyaniline-flyash composite material for shielding against electromagnetic radiation in

[4] X-band \& Ku band, AIP ADVANCES 1, 23 June 2011. 
[5] M V MURUGENDRAPPA $\dagger$, SYED KHASIM $\ddagger$ and M V N AMBIKA PRASAD*Synthesis, characterization and conductivity studies of polypyrrole-fly ash composites, Bull. Mater. Sci., Vol. 28, No. 6, October 2005, pp. 565-569. (C) Indian Academy of Sciences.

[6] R. Khan and M. Dhayal, "Chitosan/Polyaniline Hybrid Conducting Biopolymer Base Impedimetric Immunosensor to Detect Ochratoxin-A," Biosensors \& Bioelectronics,Vol. 24, 2009, pp.17001705.doi:10.1016/j.bios.2008.08.046

[7] Colaneri N F, Shacklette LW. EM1 Shielding Measurements of Conductive Polymer Blends. IEEE Trans. Instrum. Meas.41, 29 (1992).

[8] Narayan H, Alemu H, Iwuoha E. Synthesis, characterization and conductivity measurements of polyaniline and polyaniline/fly-ash composites. Phys. stat. sol. (a). 203, No. 15, 3665-3672 (2006). 